\title{
Educação Permanente como estratégia transformadora do sentido da vida do profissional de saúde frente à pandemia: Nota prévia
}

\author{
Permanent Education as a strategy to transform the meaning of life of the health professional in the
} face of the pandemic: Prior note

La Educación Permanente como estrategia para transformar el sentido de vida del profesional de la salud ante la pandemia: Nota previa

Recebido: 02/05/2021 | Revisado: 10/05/2021 | Aceito: 21/05/2021 | Publicado: 08/06/2021

\author{
Beatriz de Lima Bessa Ballesteros \\ ORCID: https://orcid.org/0000-0003-2939-1837 \\ Universidade Federal Fluminense, Brasil \\ E-mail: beatrizbessa38@gmail.com \\ Elaine Antunes Cortez \\ ORCID: https://orcid.org/0000-0003-3912-9648 \\ Universidade Federal Fluminense, Brasil \\ E-mail: nanicortez@hotmail.com
}

\begin{abstract}
Resumo
O presente estudo tem como objetivo informar sobre uma pesquisa que tem por intuito promover um espaço de açãoreflexão-ação promotor de saúde mental do profissional de saúde que atua na linha de frente do COVID-19, identificar as condições de trabalho que afetam a saúde mental dos trabalhadores de saúde, descrever o sentido da vida e estabelecer oficinas de apoio, sensibilização e diálogo sobre saúde mental e autocuidado dos trabalhadores em saúde que atua na linha de frente do COVID-19. Para tanto, o método escolhido será uma pesquisa exploratória, descritiva, com abordagem qualitativa, do tipo Pesquisa Convergente Assistencial. O cenário da pesquisa será o Centro de Tratamento Intensivo de um Centro de Referência COVID-19 na região da Baia da Ilha Grande, no Estado do Rio de Janeiro, tendo como participantes os trabalhadores de saúde do setor. Para a coleta de dados serão seguidas duas etapas, uma por meio da aplicação de um questionário semiestruturado, analisados por meio da análise de Bardin, e a segunda pela realização de oficinas baseadas na metodologia da problematização, através do uso do Arco de Maguerez. Pretende-se discutir os resultados à luz do referencial teórico de Donald Schon e Viktor E. Frankl e o referencial político através da Política Nacional de Educação Permanente em Saúde. Com esse estudo, busca-se contribuir com importante passo para estabelecer o processo de educação permanente à equipe de saúde no apoio psicossocial no atendimento ao paciente vitimizado por COVID-19.
\end{abstract}

Palavras-chave: Educação permanente; Saúde mental; Coronavírus; Trabalhadores de saúde.

\begin{abstract}
The present study aims to inform about a research that propose to provide a space of action-reflection-action that promotes mental health of the health professional who works on the front line of COVID-19, to identify the working conditions that affect the mental health of health workers, describe the meaning of life and establish workshops to support, raise awareness and dialogue on mental health and self-care of health workers who work on the front line of COVID-19. For that, the chosen method will be an exploratory, descriptive research, with a qualitative approach, of the Convergent Assistance Research type. The research scenario will be the Intensive Care Center of a Reference Center COVID-19 in the region of Baia da Ilha Grande, in the State of Rio de Janeiro, with the participation of health workers in the sector. For data collection, two stages will be followed, one through the application of a semistructured questionnaire, analyzed through Bardin's analysis, and the second through the realization of workshops based on the problematization methodology, through the use of the Maguerez Arch. We intend to discuss the results in the light of the theoretical framework of Donald Schon and Viktor E. Frankl and the political framework through the National Policy for Permanent Education in Health. With this study, we seek to contribute with an important step to establish the permanent education process to the health team in psychosocial support in caring for the victimized patient by COVID-19.
\end{abstract}

Keywords: Education continuing; Mental health; Coronavirus; Health personnel.

\section{Resumen}

El presente estudio tiene como objetivo informar sobre una investigación que tiene como objetivo promover un espacio de acción-reflexión-acción que promueva la salud mental del profesional de la salud que trabaja en la primera 
línea del COVID-19, identificar las condiciones laborales que afectan la salud mental de los trabajadores de la salud, describir el sentido de la vida y establecer talleres de apoyo, sensibilización y diálogo sobre la salud mental y el autocuidado de los trabajadores de la salud que trabajan en la primera línea del COVID-19. Para ello, el método elegido será una investigación exploratoria, descriptiva, con enfoque cualitativo, del tipo Investigación Asistencial Convergente. El escenario de la investigación será el Centro de Tratamiento Intensivo de un Centro de Referencia COVID-19 en la región de la Baia da Ilha Grande, en el Estado de Rio de Janeiro, con la participación de los trabajadores de salud del sector. Para la recolección de datos se seguirán dos pasos, uno mediante la aplicación de un cuestionario semiestructurado, analizado a través del análisis de Bardin, y el segundo mediante la realización de talleres basados en la metodología de problematización, mediante el uso del Arco de Maguerez. Pretendemos discutir los resultados a la luz del marco teórico de Donald Schon y Viktor E. Frankl y el marco político a través de la Política Nacional de Educación Permanente en Salud. Con este estudio buscamos contribuir con un paso importante para establecer el proceso de educación permanente al equipo de salud en apoyo psicosocial en el cuidado de la víctima victimizada por COVID-19.

Palabras clave: Educación continua; Salud mental; Coronavirus; Personal de salud.

\section{Introdução}

Em dezembro de 2019, foram identificados na província de Wuhan, na China, os primeiros casos de uma pneumonia de etiologia até então desconhecida, posteriormente denominada de COVID-19 (Doença de Coronavírus-2019), cujo vírus causador recebeu o nome de SARS-CoV-2 (do inglês, Severe Acute Respiratory Syndrome Coronavirus. Apesar de estudos apontarem uma origem zoonótica para o vírus, até o momento desta publicação, o reservatório animal ainda é desconhecido (Li et al., 2020; Zhu, 2020; Croda \& Garcia, 2020; Andersen et al., 2020; World Health Organization, 2021).

O vírus, altamente transmissível, se espalhou rapidamente pelo mundo, levando a Organização Mundial da Saúde (OMS) a declarar estado de pandemia em março de 2020, assim, gerando interesse internacional e levando os países a adotarem medidas de saúde pública emergenciais (Moreira et al., 2020).

A doença, potencialmente fatal, "representa o mais importante problema mundial de saúde pública dos últimos 100 anos" (Medeiros, 2020, p. 1). Em termos de saúde pública, os impactos causados pelo vírus são graves, visto sua facilidade e rapidez na propagação entre a população, levando a uma mudança abrupta nas rotinas dos serviços de saúde, observável pela intensificação das internações hospitalares em decorrência das complicações respiratórias associadas a gravidade da doença. Mediante esse cenário, a contaminação e adoecimento dos profissionais de saúde que atuam na linha de frente é uma realidade, tendo a quantidade de adoecidos se avolumado durante a pandemia (Gallasch et al., 2020; Baud et al., 2020; Cabarkapa et al., 2020).

A situação dos profissionais da saúde que estão na linha de frente do processo de cuidado, responsáveis pelo tratamento e atendimento de pacientes com COVID-19, é crítica, visto a elevada carga viral ao qual esses profissionais estão expostos, trazendo impactos, tanto à saúde física, quanto psicossocial. Assim, a carga horária exaustiva e a falta de medicamentos específicos para a cura, podem gerar significativo sofrimento mental para os mesmos (Saidel et al., 2020; SáSerafim et al., 2020).

No contexto da pandemia, é possível observar uma potencialização dos fatores que favorecem o aparecimento de situações geradoras de estresse. Esses podem estar relacionados: com a duração do período de isolamento e distanciamento social, acumulo de tarefas realizadas dentro e fora de casa, frustração e tédio, dificuldades econômicas, falta de suprimentos e informações inadequadas. Soma-se ainda a fatores correlatos a própria COVID-19, incluindo o estigma e medo de contrair a doença, a preocupação com o próprio bem estar e o de seus entes queridos, e o risco de se contaminar no trabalho, no caso dos serviços vitais (Santos et al., 2020; Van Bavel et al., 2020; Brooks et al., 2020; Li et al, 2020).

Um estudo realizado na China identificou que médicos e enfermeiros que estão na linha de frente do COVID-19, apresentam altas taxas de sintomas como: depressão, ansiedade, insônia e angústia. A pesquisa ressalta que intervenções imediatas devem ser feitas para promover o bem estar mental desses profissionais (Lai et al., 2020). 
Devido a magnitude e gravidade da pandemia de COVID-19, e os impactos psicossociais na vida dos profissionais da saúde, em decorrência da mesma, faz-se necessário gerar apoio e conhecimento, de aplicação prática imediata, com ações adaptadas e inseridas na rotina de serviços e no cotidiano de trabalho da unidade. Dessa forma, a Educação Permanente em Saúde (EPS) surge como uma estratégia educativa que permite transformar a prática profissional a partir da problematização do processo de trabalho (Portaria $n^{\circ}$ 198/GM, 2004), possibilitando, assim, reduzir o estresse através da ressignificação e do sentido da vida para o trabalho desenvolvido pelos profissionais e trabalhadores da saúde.

Este estudo justifica-se em razão da observação cotidiana como enfermeira gestora em um Centro de Referência de atendimento à COVID-19, que me permitiu recolher impressões e dados sobre a vulnerabilidade psíquica que os membros da equipe estão expostos ao lidar com uma doença instável e de conhecimentos divergentes, sobre condutas, tratamento e cura, fatores geradores de estresse e sobrecarga emocional entre os profissionais. Provocando os questionamentos sobre qual seria o caminho mais assertivo para apoio emocional aos profissionais de saúde atuantes na linha de frente da COVID-19. Sob esta perspectivas, as questões centrais que nortearão esta pesquisa são: "Qual o sentido da vida para os trabalhadores de saúde que atuam na linha de frente da COVID-19?" e "Como as condições de trabalho afetam a saúde mental dos trabalhadores de saúde?".

Dessa forma, o projeto de pesquisa possui como objetivos: Promover um espaço de ação-reflexão-ação promotor de saúde mental do trabalhador de saúde que atua na linha de frente do COVID-19; Identificar as condições de trabalho que afetam a saúde mental dos trabalhadores de saúde, descrever o sentido da vida e estabelecer oficinas de apoio, sensibilização e diálogo sobre saúde mental e autocuidado dos trabalhadores em saúde que atua na linha de frente do COVID-19.

\section{Metodologia}

Esta pesquisa está inserida no programa de Pós-graduação do Mestrado Profissional Ensino e Saúde (MPES/UFF), além de possuir anuência direta com a unidade pesquisada. Trata-se de um estudo exploratório, descritivo, com abordagem qualitativa, do tipo Pesquisa Convergente Assistencial (PCA).

A PCA tem por objetivo investigar os fenômenos ocorridos no espaço da convergência das atividades de investigação com as atividades da prática assistencial, possibilitando aos participantes analisar sua própria prática de uma forma críticoreflexiva, envolvendo pesquisadores e pesquisados na busca de estratégias para solucionar as problemáticas encontradas (Trentini et al., 2014; Bandeira, 2015).

O cenário da pesquisa será um Centro de Tratamento Intensivo (CTI) de um Centro de Referência COVID-19 situado na região da Baia da Ilha Grande, no Estado do Rio de Janeiro, tendo como participantes os trabalhadores de saúde do setor, dentre eles, médicos, enfermeiros, técnicos de enfermagem, fisioterapeutas, serviços gerais e auxiliares administrativos.

Atualmente o setor de CTI do hospital em estudo conta com 119 trabalhadores de saúde, entre equipe técnica, apoio e coordenação. Destes estão cadastrados, 23 (vinte e três) médicos intensivistas, sendo 20 (vinte) em regime de plantões 24 horas semanais e 02 (dois) médicos rotinas, 01 (um) coordenador médico, 75 (setenta e cinco) profissionais de enfermagem (enfermeiros e técnicos de enfermagem, sendo 12 (doze) enfermeiros e 60 (sessenta) técnicos de enfermagem em regime de plantões de 30 horas semanais, 01 (um) enfermeiro coordenador, 01 (um) enfermeiro rotina, 01 (um) técnico de enfermagem diarista, 15 (quinze) fisioterapeutas, sendo 14 (quatorze) em regime de plantões de 40 horas semanais e 01 (um) coordenador, 04 (quatro) trabalhadores de serviços gerais e 02 (dois) auxiliares administrativos.

Desta forma, serão convidados a participar da pesquisa os trabalhadores que fizerem parte do setor há pelo menos 6 meses, independente do regime de carga horária de trabalho ou forma de vínculo trabalhista. E, serão excluídos aqueles que estiverem afastados por motivos de férias, licenças médicas ou viagens durante o período de coleta de dados da pesquisa.

Na tentativa de extrair ao máximo as informações para conhecer as percepções e caracterizar os participantes além de 
proporcionar melhor discussão e reflexão sobre os dados, o material será coletado e analisado em duas etapas:

$1^{a}$ etapa - Será aplicado um questionário semiestruturado possuindo 03 (três) perguntas abertas a fim de identificar e esclarecer aspectos relacionados ao tema pesquisado e 07 (sete) perguntas para caracterizar a população de estudo no aspecto sociodemográfico: sexo, idade, cor/raça, estado civil e profissão. Este será apresentado a todos os participantes por meio de encontros individuais previamente acordados entre pesquisador e participante, e, somente, após a assinatura do Termo de Consentimento Livre e Esclarecido (TCLE) e do Termo de Consentimento de Imagem (TCI).

Os questionários serão analisados em duas partes, divididas entre tratamento estatístico para a análise quantitativa e a Análise de conteúdo de Bardin para tratamento das perguntas abertas, a fim de recolher da melhor forma possível impressões e elementos subjetivos relacionados à temática do estudo, permitindo, assim, apontar os tópicos a serem analisados e discutidos nas oficinas realizadas na etapa dois.

$2^{\mathrm{a}}$ etapa - Serão realizadas cinco oficinas, com o objetivo de permitir que o encontro entre os participantes se torne uma ferramenta para melhor interação e compreensão do objeto estudado, através da metodologia da problematização, através do uso das cinco etapas do Arco de Maguerez: observação da realidade e definição do problema, pontos-chave, teorização, hipóteses de solução e aplicação à realidade (Colombo \& Berbel, 2007). Os dados das oficinas serão analisados segundo a metodologia da Problematização de Berbel.

Seguindo os preceitos éticos e legais acerca para pesquisa com seres humanos, estabelecidos na Resolução do Conselho Nacional de Saúde nº 466/2012, a pesquisa foi submetida a apreciação do Comitê de Ética em Pesquisa (CEP) do Hospital Universitário Antônio Pedro da Universidade Federal Fluminense (HUAP/UFF), via Plataforma Brasil. Ademais, os trabalhadores de saúde a serem entrevistados participarão da pesquisa somente mediante a assinatura do Termo de Consentimento Livre e Esclarecido (TCLE).

\section{Resultados Esperados e Considerações Finais Parciais}

A pesquisa representará um importante passo para estabelecer o processo de educação permanente à equipe de saúde no apoio psicossocial no atendimento ao paciente vitimizado por COVID-19. Conhecer e identificar as situações em que os trabalhadores de saúde atuam irá auxiliar gestores e profissionais conhecerem precocemente o comportamento fragilizado do profissional. Portanto, com vistas ao cuidado integral e as práticas na saúde, englobando atuações assertivas, poderemos prevenir a sobrecarga emocional, minimizar riscos de morbidades psiquiátricas, utilizando técnicas relacionais tais como acolhimento e a escuta, potencializado através dos grupos de apoio.

Espera-se que a pesquisa possibilite resultados assertivos quanto a avaliação do potencial dos pontos de fragilidade dos profissionais de saúde, possibilitando a construção coletiva de estratégias e produtos significativos à prática, contribuindo com um importante passo à equipe de saúde em busca de motivação e do sentido da vida no contexto da educação permanente como estratégia transformadora frente à pandemia. Tais ações através dos profissionais de saúde serão pautadas em políticas públicas que busquem dar suporte emocional à questão do profissional da linha de frente da COVID-19, com análises sociais e psicológicas aprofundadas e voltadas para dirimir esse tipo de situação.

Tendo em vista que até o momento da publicação desta pesquisa o Brasil e o mundo estão enfrentando o SARS-CoV2, e que os profissionais da saúde que atuam na linha de frente do COVID-19 se encontram expostos a riscos de agravos físicos e emocionais decorrentes deste processo, recomenda-se que sejam realizados estudos voltados aos impactos socioeconômicos gerados pelos agravos à saúde mental desses profissionais, bem como pesquisas que apontem estratégias de enfrentamento direcionadas, tanto ao ambiente intra-hospitalar, quanto extra-hospitalar. 


\section{Referências}

Andersen, K. G., Rambaut, A., Lipkin, W. I., Holmes, E. C., \& Garry, R. F. (2020). The proximal origin of SARS-CoV-2. Nature Medicine, 26, 450-452. https://doi.org/10.1038/s41591-020-0820-9

Bandeira, C. S. (2015). Família na escola [Trabalho de Conclusão de Curso de Especialização, Universidade Federal do Rio Grande do Sul].

Baud, D., Qi, X., Nielsen-Saines, K. Musso, D., Pomar, L., \& Favre, G. (2020). Real estimates of mortality following COVID-19 infection. The Lancet Infectious Diseases, 20(7), 773. https://doi.org/10.1016/S1473-3099(20)30195-X

Brooks, S. K., Webster, R. K., Smith, L. E., Woodland, L., Wessely, S., Greenberg, N., \& Rubin, G. J. (2020). The psychological impact of quarantine and how to reduce it: rapid review of the evidence. The Lancet, 395(10227), 912-920. https://doi.org/10.1016/S0140-6736(20)30460-8

Cabarkapa, S., Nadjidai, S. E., Murgier, J., \& Ng, C. H. (2020). The psychological impact of COVID-19 and other viral epidemics on frontline healthcare workers and ways to address it: A rapid systematic review. Brain, Behavior, \& Immunity - Health, 8, 100144. https://doi.org/10.1016/j.bbih.2020.100144

Colombo, A. A.; Berbel, N. A. N. (2007). A metodologia da problematização com arco de Magarez e sua relação com os saberes dos professores. Semina: Ciências Sociais e Humanas, 28(2), 121-146. https://doi.org/10.5433/1679-0383.2007v28n2p121

Croda, J., \& Garcia, L. P. (2020). Immediate Health Surveillance Response to COVID-19 Epidemic. Epidemilogia e Serviços de Saúde, 29(1), e2020002. https://doi.org/10.5123/s1679-49742020000100021

Gallasch, C. H, Cunha, M. L., Pereira, L. A. S., \& Silva-Junior, J. S. (2020). Prevenção relacionada à exposição ocupacional do profissional de saúde no cenário de COVID-19. Revista Enfermagem UERJ, 28, e49596. https://doi.org/10.12957/reuerj.2020.49596

Lai, J., Ma, S., Wang, Y., Cai, Z., Hu, J., Wei, N., Wu, J., Du, H., Chen, T., Li, R., Tan, H., Kang, L., Yao, L., Huang, M., Wang, H., Wang, G., Liu,Z. \& Hu, S. (2020). Factors Associated With Mental Health Outcomes Among Health Care Workers Exposed to Coronavirus Disease 2019. JAMA Network Open, 3(3), e203976. https://doi.org/10.1001/jamanetworkopen.2020.3976

Li, Q., Guan, X., Wu, P., Wang, X., Zhou, L., Tong, Y., Ren, R., Leung, K. S. M., Lau, E. H. Y., Wong, J. Y., Xing, X., Xiang, N., Wu, Y., Li, C., Chen, Q., Li, D., Liu, T., Zhao, J., Lui, M., \& Feng, Z. (2020). Early transmission dynamics in Wuhan, China, of novel coronavirus-infected pneumonia. The New England Journal of Medicine, 382, 1199-1207. https://doi.org/10.1056/nejmoa2001316

Medeiros, E. A. S. (2020). A luta dos profissionais de saúde no enfrentamento da COVID-19. Acta Paulista Enfermagem, 33, e-EDT20200003. https://doi.org/10.37689/acta-ape/2020edt0003

Moreira, W. C., Sousa, A. R., \& Nóbrega, M. P. S. S. (2020). Mental illness in the general population and health professionals during COVID-19: a scoping review. Texto \& Contexto - Enfermagem, 29, e20200215. https://doi.org/10.1590/1980-265x-tce-2020-0215

Portaria no 198/GM, de 13 de fevereiro de 2004. (2004, 16 fevereiro). Instituí a Política Nacional de Educação Permanente em Saúde como estratégia do Sistema Único de Saúde para a formação e o desenvolvimento de trabalhadores para o setor e dá outras providências.

Sá-Serafim, R., Do Bú, E., \& Lima-Nunes, A. (2020). Manual de Diretrizes para Atenção Psicológica nos Hospitais em Tempos de Combate ao COVID-19. Revista Saúde e Ciência Online, 8(2), 1-44. https://rsc.revistas.ufcg.edu.br/index.php/rsc/article/view/401/385

Saidel, M. G. B., Lima, M. H. M., Campos, C. J. G., Loyola, C. M. D., Espiridião, E., \& Rodrigues, J. (2020). Intervenções em saúde mental para profissionais de saúde frente a pandemia de Coronavírus. Revista Enfermagem UERJ, 28, e49923. https://doi.org/10.12957/reuerj.2020.49923

Santos, B. G., Lira, A. V. A. A., Mattos, M. S. B., \& Pachú, C. O. (2020). Estratégias para redução do estresse ocupacional em trabalhadores da saúde durante a pandemia por COVID-19: uma revisão da literatura. Research, Society and Development, 9(11), e1639119707. https://doi.org/10.33448/rsd-v9i11.9707

Trentini, M., Paim, L., \& Silva, D. M. G. V. (2014). Pesquisa Convergente Assistencial: delineamento provocador de mudanças nas práticas de saúde (3a ed). Moriá.

Van Bavel, J. J., Baicker, K., Boggio, P. S., Capraro, V., Cichocka, A., Cikara, M., Crockett, M. J., Crum, A. J., Douglas, K. M., Druckman, J. N., Drury, J., Dube, O., Ellemers N., Finkel, E. J., Fowler, J. H., Gelfand, M., Han, S., Haslam, A., Jetten, J., \& Willer, R. (2020). Using social and behavioural science to support COVID-19 pandemic response. Nature Human Behaviour, 4, 460-471. https://doi.org/10.1038/s41562-020-0884-z

World Health Organization. (2021). WHO-convened global study of origins of SARS-CoV-2: China Part. https://www.who.int/publications/i/item/whoconvened-global-study-of-origins-of-sars-cov-2-china-part

Zhu, N., Zhang, D., Wang, W., Li, X., Yang, B, Song, J., Zhao, X., Huang, B., Shi, W., Lu, R., Niu, P., Zhan, F., Ma, X., Wang, D., Xu, W., Wu, G., Gao, G. F., \& Tan, W. (2020). A Novel Coronavirus from Patients with Pneumonia in China, $2019 . \quad N \quad$ Engl J Med, $382,727-733$. https://doi.org/10.1056/NEJMoa2001017 